3rd.-Passed a good night; no pain nor tenderness; felt hungry; took some fish. Bandage and dressings removed; wound looked healthy, with a slight discharge from the lower end of it; bread poultices applied. Towards evening she had pain and spasm at the chest, which were relieved by ether and ammonia.

4th. - Slept well, and expressed herself as very much better; very little pain; wound looking well, and discharge increasing.

5th.-Had less pain; pulse 120; tongue furred; wound healthy, and discharges freely a grumous-looking matter (which Mr. Adams believes to be broken-up placenta), the cord having come away in the poultices. Took six ounces of sherry wine, beef-tea, and fish; often feels very hungry.

6th. - Passed a good night as usual; tongue rather dry. The bowels not having been relieved since the operation, Mr. Adams ordered two drachms of castor oil, which operated very freely.

7th.-Took some jelly, and a chop; wine increased to eight ounces, none of the ligatures having come away. The whole length of the wound had now healed by the first intention, with the exception of the lower end, where there is a sinus, of the size of a threepenny-piece, out of which the ligatures hang, and the discharge freely escapes.

8th.-The sutures were now beginning to irritate the bond of union. Mr. Payne removed them. Her pulse sank to 100 .

10th. - Continues to improve.

12th. - Mr. Adams removed some of the ligatures, when the discharge of about half an ounce of offensive grumous fluid fol. lowed.

17th. - Continues to improve in every respect.

19th. - Mr. Adams removed the remainder of the ligatures, and there again ensued the same offensive discharge. He has ordered her to sit up for a few hours daily.

It is now the twentieth day since the operation. She takes four ounces of wine, and a pint of porter, with a chop, daily, and has not the slightest feeling of uneasiness, except from the annoyance of the discharge, which is, however, slowly decreasing

July 11th. - She is still going on well, gets up, and walks about the ward daily; but there are no signs of a placenta or membrane coming away, although the offensive discharge con. tinues.

\section{KING'S COLLEGE HOSPITAL.}

ANEURISM OF THE ABDOMINAL AORTA, PRODUCING EROSION OF THE VERTEBRE; FATAL RESULT SLOWLY INDUCED BY GRADUAL HAMORRHAGE BEHIND THE PERITONEUM.

(Under the care of Dr. Johnson.)

OF the various kinds of tumours which arise in the abdomen, perhaps there are none of more serious import than aneurisms, whether springing direct from the aorta or from some one of its numerous branches. If the sac should burst into the peritoneal cavity, death very quickly ensues. On the other hand, should the aneurism open into the cellular tissue without or externally to the peritoneum, the extravasation of blood goes on slowly, and a fatal result will gradually take place. This form of extra. peritoneal hæmorrhage has been known to give rise to pulsating tumours in the iliac, hypochondriac, and lumbar regions.

The slowness of death in the following case is clearly accounted for by the gradual hæmorrhage which occurred between the peritoneal layers of the transverse and ascending mesocolon, and which was found to extend to the cellular tissue of the pelvis. The situation of the tumour was below the origin of the superior mesenteric artery, where it had already produced erosion of the bodies of the contiguous vertebra. As might be expected, its pressure on the vena cava caused the prominence of the abdominal and the varicosity of the superficial crural veins; whilst the albuminuria was wholly attributable to the obstruction of the left renal vein from the same cause. It is extremely probable that the paroxysmal pain denoted a rupture of the aneurismal coats; but from the circumstance of the blood escaping between the layers of the meso-colon, it quickly coagulated, and impeded further flow.

For the notes of the following case we are indebted to Dr. A. Ernest Sansom, house-physician to the hospital :-

Henry $\mathrm{P}_{-}$- , a carpenter, aged thirty-eight, was admitted May 18th, 1860. He has had to lift heavy weights constantly; had no serious illness till three months before admission, when a sudden and severe pain occurred one morning as he was going to his work, and caused him to return bome. The pain was between the hypochondria; it passed off, but recurred at in. tervals. He suffered besides from pain in the back. Some time afterwards he felt a throbbing at the pit of the stomach, which he thought to be palpitation of the heart. Coincident with these symptoms were general malaise, sickness after meals, and considerable loss of flesh. Latterly he passed but small quantities of urine, and the pain in the back was increased by micturition.

On admission, he complained of pain in the back, varying in severity, causing him frequently to change his position, but not increased by pressure. He presented the appearance of a strong, healthy man, who had been debilitated by disease. The superficial veins of both legs were seen to be varicose, and those of the abdomen were tortuous and enlarged, especially on the right side. From the ensiform cartilage to within an inch of the umbilicus was felt a distinctly pulsating tumour, which communicated a thrill to the fingers and a rasping bruit to the ear, synchronous with the pulss at the wrist. A small, movable swelling was felt in front of the tumour. The bruit was heard, though somewhat faintly, behind on either side of the spine, chiefly on a level with the ninth dorsal vertebra. There was an exaggerated curve in the lumbar region, and the ribs bulged very much. Breathing pure; heart sounds natural; bruit not heard above the diaphragm; pulse 62 . The urine was albuminous; specific gravity 1018, of a rather deep sherry colour, containing some small waxy casts. Ordered a compound soap pill and compound magnesia mixture twice a day; soap plaster to the back.

The pains were relieved, and he continued better until the 24th, when, about six 1.M., immediately after he had had an evacuation, he had an epileptic fit. After emerging from this, he fell into paroxysms, to all appearance, of violent pain, to such extent that it required three men to hold him. An enema of warm water was first administered, and then, soon after the commencement of the muscular contortions, Dr. Sansom administered chloroform. Just previously, the pulsation of the tumour was found to be diminished, and pressure over its situation seemed to cause pain. Chloroform soon brought about a perfect calm, which continued for half an hour, when it was re-administered; and so, at intervals, throughout the night. At half-past six the following morning the patient was sensibly calmer. At ten A.M. forty minims of solution of acetate of morphia were given by the mouth, which failed, however, to produce the desired effect, so chloroform was continued, but at longer intervals. At two P.M. twenty minims of solution of acetate of morphia were injected beneath the skin of the right upper arm, which soon produced a manifest and continued quieting effect. Soon the paroxysms ceased. On the 26 th he had a threatening of their return; but morphia was administered, and he became quiet. The tumour was now felt to pulsate over a wider area. The bowels were relieved by an enema.

On the 31st the patient died. No change was noticed till two hours before death, when the face became pale and gradually completely blanched; the heart's beats were very feebly felt, and after a short time were imperceptible. Occasionally, however, after all pulsation had ceased, a sigh was heaved.

At the post-mortem examination, an aneurismal tumour, of about the size of two fists, was seen to involve the abdominal aorta below the origin of the superior mesenteric artery. The aneurism had burst between the layers of the peritoneum in the transverse and ascending meso-colon, the blood being traced downwards to the cellular tissue of the pelvis. The bodies of the last dorsal and first two lumbar vertebræ had been much eroded, the intervertebral substances standing out nearly a quarter of an inch from the corresponding vertebræ. The small swelling felt in front of the tumour was found to be the pylorus. The aneurism had stretched the vena cava on its surface, and the left renal vein was flattened by it to a ribandlike band. The kidneys, especially the left, were enlarged and congested.

\section{UNIVERSITY COLLEGE HOSPITAL.}

SEQUEL TO A CASE OF EMBOLON IN THE CEREBRAL ARTERIES, WHICH HAD PRODUCED HEMIPLEGIA, THE RESULT OF RHEUMATIC ENDOCARDITIS.

(Under the care of Dr. JENNER.)

MANY physicians are sceptical as to the possibility of diagnosing the existence of embolon during life, and it therefore becomes a matter of importance to give the sequel to any case that we have thought of sufficient interest to record in our "Mirror." Of three instances which appeared in the first volume of this journal for the present year (pp. 466-7), the 